\title{
A sexually dimorphic peptidergic system in the lower spinal cord controlling penile function in non-human primates
}

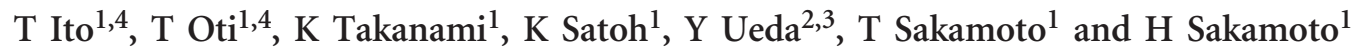

Study design: Experimental animal study.

Objectives: Although a population of gastrin-releasing peptide (GRP) neurons in the lumbar spinal cord has an important role in erection and ejaculation in rats, little information exists on this GRP system in primates. To identify the male-specific GRP system in the primate spinal cord, we studied the lumbosacral cord in macaque monkeys as a non-human primate model.

Setting: University laboratory in Japan.

Methods: To determine the gene sequence of GRP precursors, the rhesus macaque monkey genomic sequence data were searched, followed by phylogenetic analysis. Subsequently, immunocytochemical analysis for GRP was performed in the monkey spinal cord.

Results: We have used bioinformatics to identify the ortholog gene for GRP precursor in macaque monkeys. Phylogenetic analysis suggested that primate prepro-GRP is separated from that of other mammalian species and clustered to an independent branch as primates. Immunocytochemistry for GRP further demonstrated that male-dominant sexual dimorphism was found in the spinal GRP system in monkeys as in rodents.

Conclusion: We have demonstrated in macaque monkeys that the GRP system in the lower spinal cord shows male-specific dimorphism and may have an important role in penile functions not only in rodents but also in primates.

Sponsorship: Tissues of Nihonzaru (Japanese macaque monkeys) were provided in part by National Institutes of Natural Sciences (NINS) through the National Bio-Resource Project (NBRP) of the MEXT, Japan. This work was supported in part by KAKENHI from the Japan Society for the Promotion of Science (JSPS) (to KT; 15KKO343, 15J40220 and HS; 15K15202, 15KK0257, 15H05724). Spinal Cord (2018) 56, 57-62; doi:10.1038/sc.2017.105; published online 12 September 2017

\section{INTRODUCTION}

Severe injuries in the lower spinal cord frequently cause sexual dysfunction in men, including erectile dysfunction (ED) and ejaculation difficulties. ${ }^{1,2}$ This indicates that important neural control circuits for penile function are located within the lower spinal cord. ${ }^{3-9}$ We previously demonstrated that a population of gastrin-releasing peptide (GRP) neurons in the lumbar spinal cord has an important role in penile erection and ejaculation in rats. ${ }^{10,11}$ This system of GRP neurons is sexually dimorphic, being prominent in male rats but vestigial or absent in female rats. ${ }^{10-12}$ Recently, we reported that this sexually dimorphic system has been identified not only in rodents but also in the Asian house musk shrew (Suncus murinus) (order of Eulipotyphla; formerly Insectivora). ${ }^{13}$ These results suggest that the sexually dimorphic spinal GRP system is general in mammals. However, little information exists on the spinal GRP system controlling male sexual function in primates, although it is clinically important to know whether the GRP system exists and has similar functions in the lower spinal cord in primates. Macaque monkeys appear to be an excellent model because they are close primate relatives to humans. ${ }^{14}$ Therefore, in this study, we worked to identify the sexually dimorphic spinal GRP system in primates using the Japanese macaque monkey (Macaca fuscata).

\section{METHODS}

Experimental animals

Male and female Japanese macaque monkeys, M. fuscata $(n=3: 3-, 7$ - and 9-year-old males, weight 2.9-9 kg; $n=3: 8-, 10$ - and 11-year-old females, $7-8.3 \mathrm{~kg}$ ), were used in this study. Monkeys were maintained in a temperaturecontrolled $\left(22-24^{\circ} \mathrm{C}\right)$ room under a daily photoperiod of $12 / 12 \mathrm{~h} \mathrm{light/dark}$ cycle (lights on 0800 hours-2000 hours). We confirmed these animals were free of specific pathogens. Food and water were available ad libitum. All animals were kept in individual cages. The experimental protocols followed the guideline of the Ministry of Education, Culture, Sports, Science and Technology (MEXT) of Japan and were approved in accordance with the Guide for the Care and Use of Laboratory Animals prepared by Okayama University (Okayama, Japan), by Kyoto Prefectural University of Medicine (Kyoto, Japan) and by Kansai Medical University (Osaka, Japan). All efforts were made to minimize animal suffering and reduce the number of animals used in this study.

\section{Gene database search and phylogenetic analysis}

To evaluate the gene sequence of GRP precursors, the rhesus macaque monkey (M. mulatta) genomic sequence data were searched on the NCBI

${ }^{1}$ Ushimado Marine Institute (UMI), Graduate School of Natural Science and Technology, Okayama University, Setouchi, Japan; ${ }^{2}$ Department of Physiology, Kyoto Prefectural University of Medicine, Kyoto, Japan and ${ }^{3}$ Department of Physiology, Kansai Medical University, Hirakata, Japan

${ }^{4}$ These authors contributed equally to this work.

Correspondence: Dr H Sakamoto, Ushimado Marine Institute (UMI), Graduate School of Natural Science and Technology, Okayama University, 130-17 Kashino, Ushimado Okayama, Setouchi 701-4303, Japan.

E-mail: hsakamo@okayama-u.ac.jp

Received 18 May 2017; revised 18 July 2017; accepted 19 July 2017; published online 12 September 2017 
(www.ncbi.nlm.nih.gov) DNA database by blastn using the human nucleotide sequences of the precursors of GRP as queries. On the basis of the monkey genomic sequence data, the deduced amino acid residues were compared with those of other mammalian species.

Alignment of the amino acid sequences of the GRP precursor protein from different species and human neuromedin B as out-group was performed using the ClustalW sequence alignment program. Phylogenetic trees were constructed using the neighbor-joining method and viewed with TreeView (Version 1.6.6) (http://jtreeview.sourceforge.net/). Details of the program settings are given in the legend for Figure 1.

\section{Tissue preparation}

Monkeys (males, $n=3$; females, $n=3$ ) were anesthetized with an overdose of pentobarbital sodium $\left(90 \mathrm{mg} \mathrm{kg}^{-1}\right.$ i.m.) and transcardially perfused with physiological saline followed by $4 \%$ paraformaldehyde in $0.1 \mathrm{M}$ phosphate buffer (PB) ( $\mathrm{pH}$ 7.4). Lumbosacral spinal cords were quickly removed and immersed in the same fixative overnight at $4{ }^{\circ} \mathrm{C}$. After immersion in $25 \%$ sucrose in $0.1 \mathrm{M} \mathrm{PB}$ at $4{ }^{\circ} \mathrm{C}$ for cryoprotection until they sank, the preparations were quickly frozen using powdered dry ice and cut into $30 \mu \mathrm{m}$ cross-sections using a cryostat (CM3050 S, Leica, Nussloch, Germany) and stored in a cryoprotectant (30\% glycerol and 30\% ethylene glycol in phosphate-buffered saline; PBS) (pH 7.4) at $-20^{\circ} \mathrm{C}$ until use.

\section{Immunocytochemistry and immunofluorescence}

We performed an immunocytochemical analysis according to our established methods using cryosections. ${ }^{10,11,13}$ In brief, tissue sections were first rinsed five times with PBS to remove the cryoprotectant. Endogenous peroxidase activity

a

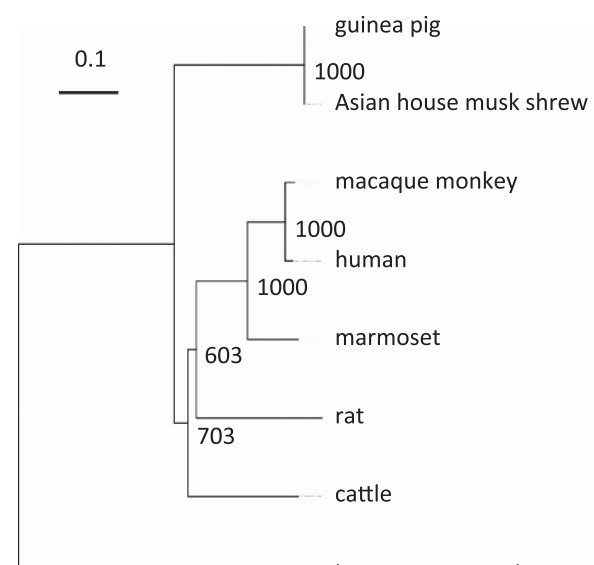

b

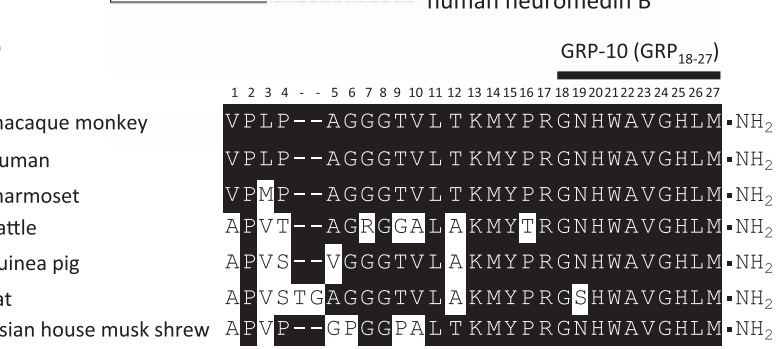

Figure 1 The evolutionary relationships of primate GRP with other mammalian species. (a) A phylogenetic tree comprising prepro-GRP was constructed using the neighbor-joining method. Branch lengths are proportional to the number of amino acid changes on the branch. The values represent bootstrap scores for 1000 trials, indicating the credibility of each branch. Horizontal bar indicates the genetic distance. (b) A comparison of the amino acid sequences of Macaca GRP with the corresponding sequences of mature GRP in mammals. Residue identity to Macaca GRP denoted by the highlight. Residue deletions denoted by ' - ' are inserted into some sequences to maximize structural similarity. Line-highlighted sequence indicates that the mature GRP in monkeys is identical to human GRP. was eliminated by incubation in $1 \% \mathrm{H}_{2} \mathrm{O}_{2}$ in absolute methanol for $20 \mathrm{~min}$ followed by three 5-min rinses with PBS. This $\mathrm{H}_{2} \mathrm{O}_{2}$ treatment process was omitted in the immunofluorescence method. After blocking nonspecific binding with $1 \%$ normal goat serum and $1 \%$ BSA in PBS containing $0.3 \%$ Triton X-100 for $30 \mathrm{~min}$ at room temperature, sections were incubated with primary rabbit antiserum, which recognizes a 10 -amino acid peptide called neuromedin C or GRP-10 (11081-05015; AssayPro, St Charles, MO, USA) (1:2000 dilution) for 3 days at $4{ }^{\circ} \mathrm{C}$. The GRP antiserum used in this study has previously been shown to be specific for GRP in the spinal cord of rats, mice and Asian house musk shrews. ${ }^{13,15-17}$ Immunoreactive products were detected with a streptavidin-biotin kit (Nichirei, Tokyo, Japan), followed by diaminobenzidine (DAB) development according to our previous method. ${ }^{10,18}$ GRP immunoreactivities in the spinal cord were localized using an FSX100 microscope (Olympus, Tokyo, Japan). In addition, GRP-containing fibers were visualized with an immunofluorescence method using Alexa Fluor 488-linked anti-rabbit IgG raised in goats (1:1000 dilution) (Molecular Probes, Eugene, OR, USA). To determine the projection site of GRP-containing fibers, doubleimmunofluorescence staining for GRP (1:1000 dilution) and neuronal nitric oxide synthase (1:5000 dilution) (nNOS; A-11; a mouse monoclonal antibody raised against amino acids 2-300 of nNOS of human origin, Santa Cruz Biotechnology, Santa Cruz, CA, USA), a marker protein for neurons in the sacral autonomic nucleus (SAN), was performed in a similar way described previously in rats, mice and Asian house musk shrews. ${ }^{10,11,13}$ Alexa Fluor 546linked anti-mouse IgG (Molecular Probes) and Alexa Fluor 488-linked antirabbit IgG, both raised in goats, were used at a 1:1000 dilution for detection. Immunostained sections were imaged with a confocal laser scanning microscope (FluoView FV1000, Olympus). Immunocytochemistry studies were repeated independently at least three times using different animals and they produced similar results.

\section{Antibody specificity}

The rabbit polyclonal GRP antiserum was raised against 10-amino acid GRP-10 consisting of residues 20-29 of the full rat GRP. This antiserum produced identical patterns of labeling in the distribution of GRP-positive fibers in the lumbosacral spinal cord as that achieved by immunocytochemistry using the same $e^{15,16}$ or other antisera ${ }^{10,19}$ in rats. Control procedures for the DAB method were performed using pre-absorption of the working dilution (1:2000) of the primary antiserum with saturating concentration of monkey $\mathrm{GRP}_{18-27}$ (or GRP-10; corresponding to the monkey GRP deduced amino acid residues from the genomic information, see Figure 1a) antigen peptide, Gly-Asn-His-Trp-AlaVal-Gly-His-Leu-Met (50 $\mathrm{g} \mathrm{ml}^{-1}$, produced in AnaSpec; San Jose, CA, USA) for $1 \mathrm{~h}$ at room temperature before use. The GRP-positive fibers were detected according to the above protocol for peroxidase immunocytochemistry.

\section{Semi-quantitative analysis}

We performed a semi-quantitative analysis of GRP expression by using the DAB-stained immunoreacted sections. To determine the density of GRPpositive fibers in the lumbosacral spinal cord (L5-S1 level), at least five crosssections $(30-\mu \mathrm{m}$ thick) per animal were randomly selected, and the digital images of two regions (the SAN and dorsal horn $(\mathrm{DH})$ ) were prepared (magnification, $\times 200)$. The unit area $\left(343 \times 469 \mu \mathrm{m}^{2}\right)$ was analyzed to localize the nuclei at the center of each area. The optical density of GRP staining (DAB) was determined using black-and-white images that were converted from micrographs using ImageJ software (ImageJ 1.44p; National Institutes of Health, Bethesda, MD, USA) according to our established methods. ${ }^{10,11,18,20}$ Briefly, the optical density of background labeling was estimated by comparisons with similar areas of the control sections reacted with the anti-GRP antiserum that was incubated first with an excess of peptide antigen $\left(50 \mu \mathrm{g} \mathrm{ml}^{-1}\right)$. GRP expression was undetectable in these sections. Each threshold optical density was determined by normalizing the data to those of the pre-absorbed sections. The GRP-positive fiber pixel density was semiquantified as the average pixel density in the SAN and DH of each animal. Micrographs were coded and evaluated without the knowledge of the experimental group designation, and the code was not broken until the analysis was complete. Data are expressed as the relative value against the mean of the $\mathrm{DH}$ value in males. 


\section{Statement of ethics}

We certify that all applicable institutional and governmental regulations concerning the ethical use of animals were followed during the course of this research.

\section{RESULTS}

\section{Sequence analysis of the monkey GRP precursor gene}

To investigate the evolutionary relationships of primate GRP with other mammalian species, a phylogenetic tree based on the deduced amino acid sequence of prepro-GRP was constructed using the neighbor-joining method (Figure 1a). According to the tree, monkey prepro-GRP at the amino acid level was grouped in the primate branch and separated from that of rodents and other mammalian species (Figure 1a). In addition, the sequence in common marmoset, a new world monkey (Platyrrhini), was separated from that in old world monkeys (Catarrhini) and possibly proximal in the primate branch in terms of prepro-GRP (Figure 1a). According to the deduced amino acid sequence, the macaque monkey's prepro-GRP starts with a signal peptide, followed by the bioactive $\mathrm{GRP}_{1-27}$ (mature GRP) including the GRP-10 motif at the C terminus of mature GRP, which is highly conserved in mammals (Figure 1b). The mature GRP in monkeys is identical to human GRP (Figure 1b).
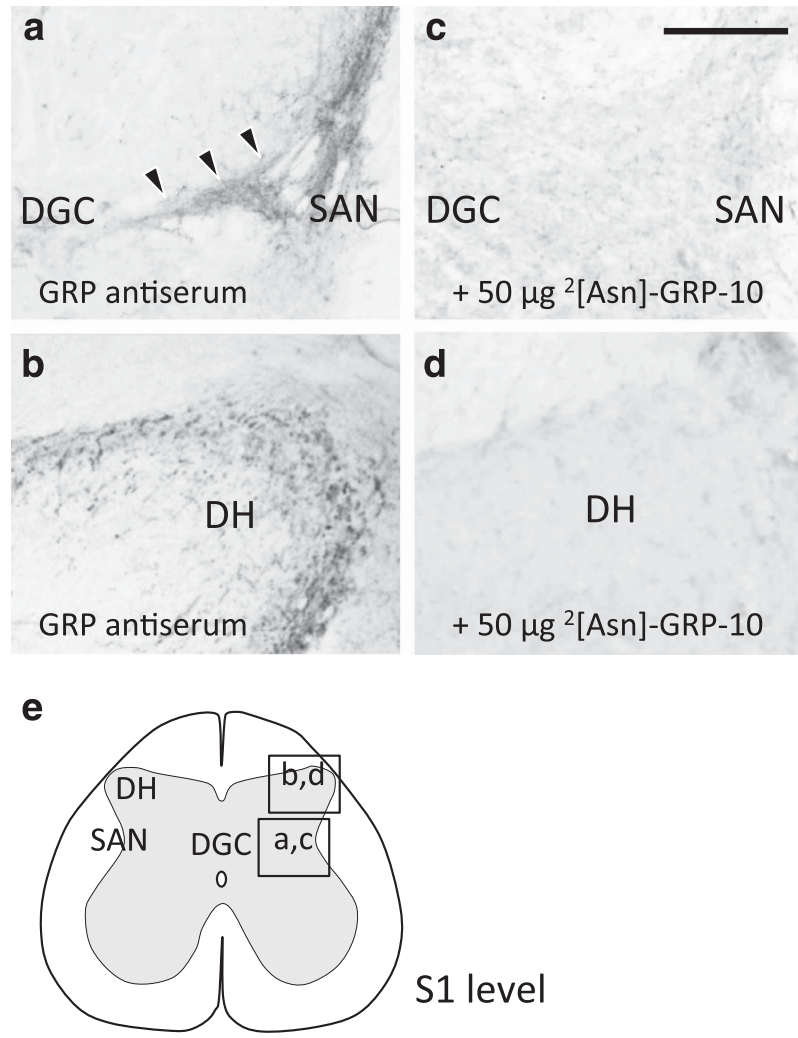

Figure 2 Immunocytochemistry analysis of GRP in the sacral spinal cord of Japanese macaque monkeys. GRP-containing fibers in the sacral spinal cord (S1 level) were present in the DGC (a), SAN (a) and spinal DH (b) in monkeys. GRP-containing fibers projected toward the SAN is obvious (arrowheads in a). Preabsorbing the working dilution (1:2000) of the primary GRP antiserum with a saturating concentration of GRP antigen peptide (GRP-10; $50 \mu \mathrm{g} \mathrm{ml}^{-1}$ ) overnight at $4^{\circ} \mathrm{C}$ before use eliminates the staining in the monkey spinal cord (c, d). (e) Schematic drawing of the sacral spinal cord (S1 level). Scale bar $=200 \mu \mathrm{m}$.
GRP in the lumbosacral spinal cord of Japanese macaque monkeys Immunocytochemical detection of GRP in the lumbosacral spinal cord (L5-S1 level) of male monkeys was performed. In the monkey lumbar and sacral cords, GRP-containing fibers were found in the dorsal gray commissure (DGC) (Figure 2, S1 level), as in rats and mice. ${ }^{10,13,18}$ Furthermore, GRP-containing fibers appeared to project toward the SAN (arrowheads in Figure 2a). We also found GRP fibers in the spinal DH of monkey lumbar and sacral cords (Figure 2b, S1 level). Use of the pre-absorbed antibody abolished immunostaining $(n=3 ; 2$ males and 1 female) (Figures $2 c$ and $d$ ). We then examined the sexually dimorphic distribution of GRP-containing fibers in the SAN of the monkey sacral cord because SAN provides autonomic preganglionic fibers to the genitalia (Figure 3). ${ }^{21,22} \mathrm{We}$ found male-dominant projection of GRP-positive fibers was obvious in the transition area between DGC and SAN (Figures 3a-d). Double immunofluorescence for GRP and nNOS, a marker for autonomic preganglionic neurons, ${ }^{23}$ clearly showed a strong projection of GRP-positive fibers into the SAN in males but only few fibers in females (Figures $3 e$ and f). Double immunofluorescence for GRP and nNOS showed that GRP-positive fibers in the SAN were more prominent in male than in female monkeys (Figures $3 \mathrm{~g}-\mathrm{j}$ ). Semi-quantitative analyses also supported this sex difference in the SAN but not DH (Figures $3 \mathrm{k}$ and $\mathrm{l}$ ). In addition, our GRP immunocytochemistry did not demonstrate GRP neuronal somata in the macaque spinal cord.

\section{DISCUSSION}

The aim of this study was to demonstrate, in a model primate, the sexually dimorphic GRP system in the spinal cord controlling penile function. To our knowledge, this is the first demonstration of this system using Japanese macaque monkeys, M. fuscata.

Phylogenetic analysis suggested that primate prepro-GRP is separated from that of other mammalian species and clustered to an independent branch as primates. Macaque (Catarrhini) and human prepro-GRP are very closely related, and also related to that of marmoset (Platyrrhini), but rather different from that of rat, and even more distinct from that of Asian house musk shrew (S. murinus) (Figure 1b). Human $\mathrm{GRP}_{18-27}$ (or $\mathrm{GRP}_{20-29}$ in mice and rats) is a possible C-terminal fragment of mature GRP and was identified from porcine spinal cord originally as neuromedin C. ${ }^{24}$ A more appropriate name is perhaps GRP- $10 .{ }^{25}$ It has been reported in rodents that the second amino acid of GRP-10 is a serine, ${ }^{13,26}$ but an asparagine exists in this location in most other mammals including humans, ${ }^{27}$ cattle, ${ }^{28}$ pigs, ${ }^{29}$ sheep, ${ }^{30} \operatorname{dogs}^{31}$ and guinea pigs. ${ }^{32}$ In this study, we found that the deduced second amino acid of GRP-10 is an asparagine in macaque monkeys and identical to those of many mammals other than rodents. ${ }^{13,26}$ Moreover, the amino acid sequence of entire mature GRP is identical in humans and monkeys. Taken together, these results demonstrate that the GRP gene shows a high homology especially among old world monkeys and, furthermore, is well conserved among primates.

Using the Japanese macaque monkey as a non-human primate model, we demonstrated immunocytochemically that GRP-containing fibers projecting into the SAN of this primate show a remarkable male-dominant sexual dimorphism similar to that in rodents. ${ }^{10,11,13,18}$ The SAN provides autonomic preganglionic fibers to the genitalia. ${ }^{21,22}$ Spinal GRP-positive axons have also been shown to make direct synaptic contacts with somatic motor neurons of the spinal nucleus of bulbocavernosus (SNB) located in the sacral spinal cord in male rats, ${ }^{33,34}$ and GRP-sensitive receptors are expressed by rat SNB motor neurons. ${ }^{10,25}$ The SNB in rats, which innervates the striated perineal muscles attached to the base of the penis, ${ }^{9,35-37}$ is homologous with 

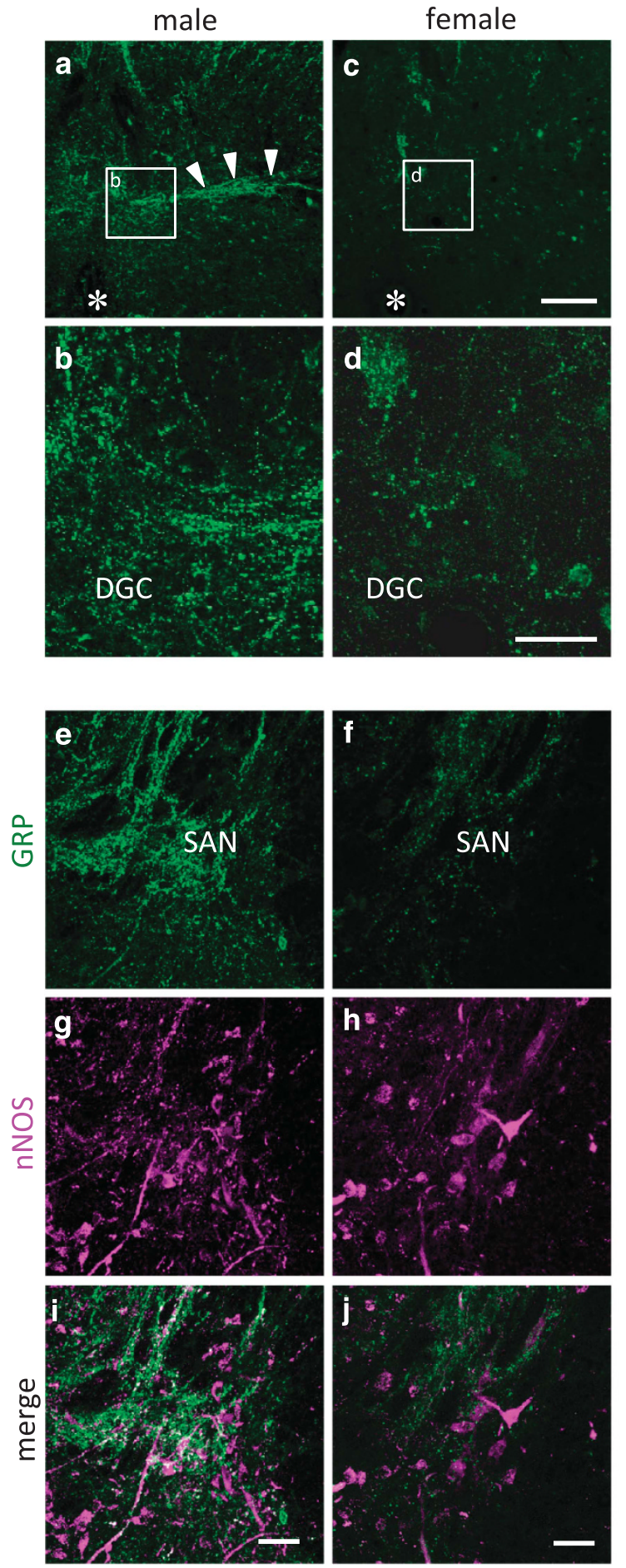
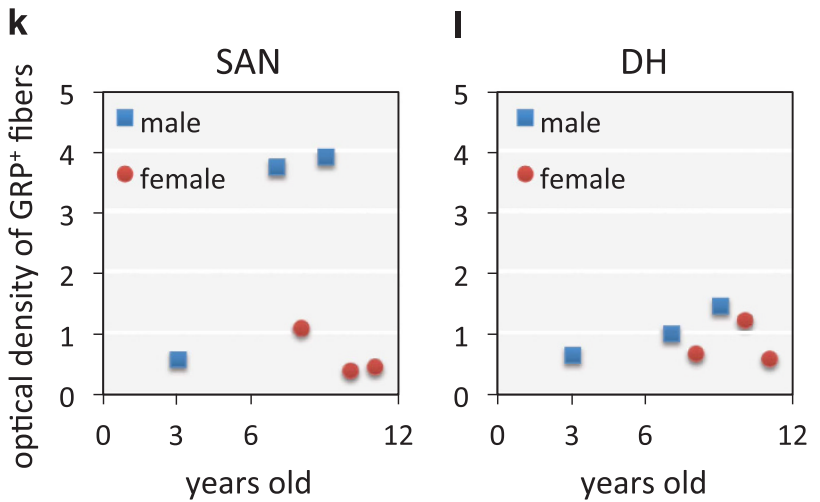

m

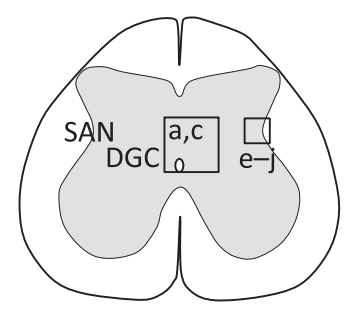

S1 level

Figure 3 Immunocytochemistry analysis for GRP in the sacral spinal cord (S1 level) of Japanese macaque monkeys. Male-specific expression for GRP is observed in the monkey sacral spinal cord (a, b). Possible GRP-containing fibers projected toward the SAN are male-specific (a, $\mathbf{c}$ ), and the male-dominant expression is also observed in the DGC (b, d). Asterisks $\left(^{*}\right)$ indicate the location of the central canal in $\mathbf{a}, \mathbf{c}$. GRP-containing fibers (green) are obviously observed in males (e) but weak in females (f). The nNOS serves as a marker for SAN neurons, and the immunoreactivity for nNOS in the SAN neurons (magenta) showed no sex difference $(\mathbf{g}, \mathbf{h})$. Double immunofluorescence for GRP and nNOS revealed closely appositions of GRP-containing fibers with the cell bodies and proximal dendrites of nNOS-positive neurons in the SAN (i, j). Semi-quantitative analysis suggests that the intensity of GRP-positive fibers in the sacral spinal cord is greater in males than in females in the autonomic centers of the SAN (k) but not in the somatic sensory layers of the spinal DH (I). Schematic drawing of the sacral spinal cord (S1 level) (m). Scale bars $=(\mathbf{c}) 200 \mu \mathrm{m} ;(\mathbf{d}, \mathbf{i}, \mathbf{j}) 50 \mu \mathrm{m}$.

the human Onuf's nucleus, a sexually dimorphic nucleus located in the motor neuron pools of the sacral spinal cord that has an important role in the micturition and defecatory as well as in rhythmic contractions of perineal muscles during orgasm. ${ }^{38}$ The number of motor neurons in Onuf's nucleus in humans is greater in men than in women. ${ }^{35,38-40}$ Taken together with the behavioral results from our 
rodent studies, ${ }^{10}$ our present results in a model primate strongly suggest that the sexually dimorphic spinal GRP system in primates is involved in the regulation of both autonomic and somatic penile functions. Future studies focusing on GRP receptors in the monkey spinal cord could provide new insights into the peptidergic control circuit for penile functions in primates.

Although our immunocytochemical procedure using formaldehydefixed tissues did not demonstrate GRP neuronal somata in the present study, when taken together with many studies in rodents, ${ }^{10,18}$ it is likely that monkey GRP neurons are also located in the lumbar spinal cord. Intraspinal or intrathecal administration of colchicine could be used to facilitate the immunocytochemistry; alternatively, gene expression analyses of fresh monkey tissues could be used to localize the somata of monkey spinal GRP neurons.

GRP-containing neurons in the lumbar spinal cord highly coexpress androgen receptors in rats ${ }^{10}$ and mice, ${ }^{13}$ and the sexually dimorphic expression of GRP in the lower spinal cord is also controlled and maintained in an androgen-dependent manner in rodents. ${ }^{11,12,18}$ A 3-year-old male monkey (possibly pre-puberty) was also used in this study. The lower optical density of GRP-positive fibers in this animal is consistent with the finding ${ }^{20}$ that the androgen surge during male puberty has an important role in the development of the male-specific GRP function in the monkey spinal cord. Thus, expression of androgen receptors would be expected to be an important characteristic of the GRP system in monkeys. Future attention should therefore be focused on the co-localization of androgen receptor/GRP in the monkey spinal cord.

Our immunocytochemical study also located GRP-immunoreactive fibers in the spinal DH of the macaque monkeys, but, unlike those in the SAN, they were not sexually dimorphic and, by analogy with our studies in rodents, were probably derived from dorsal root ganglion GRP neurons involved in itch. ${ }^{15,41}$

Clinical data show that ED is an increasingly common condition predicted to affect more than 300 million men worldwide by 2025. ${ }^{42,43}$ The increasing rates may reflect the increasing stress in society and could contribute to declining the birth rate that is becoming a serious social problem in certain societies. ${ }^{43}$ It has long been established that $\mathrm{ED}$ is a multi-factorial dysfunction, but most treatments, including sildenafil citrate, have targeted penile vasculopathy. ${ }^{44}$ Current treatments can have unwanted side effects and, importantly, fail to address the underlying neural control problem. Our finding that the GRP system controlling erection and ejaculation in rodents ${ }^{10}$ is also present in primates could, in the future, provide new therapeutic approaches to sexual problems in men.

\section{CONCLUSIONS}

Here we demonstrate, using Japanese macaque monkeys as a model non-human primate, that the GRP system in the lower spinal cord shows a male-specific dimorphism and is likely to have an important role in penile functions in primates. The sexually dimorphic spinal GRP system controlling penile functions appeared to be general in primates, which could bridge to therapeutic treatments for sexual problems in men.

\section{DATA ARCHIVING}

There were no data to deposit.

\section{CONFLICT OF INTEREST}

The authors declare no conflict of interest.

\section{ACKNOWLEDGEMENTS}

We are grateful to Prof John F Morris (University of Oxford, UK) for his valuable discussion and for reading this manuscript. We thank Prof Mitsuhiro Kawata, Prof Minoru Kimura, Prof Kazuhiro Yagita, Prof Masaki Isoda and Prof Kae Nakamura for providing the fixed monkey spinal cords, their encouragement and/or critical discussion of this study. We thank Dr Yasuhisa Kobayashi, Mr Kazuhiro Saito, Mr Kei Tamura and Mr Toshitsugu Takahashi for their technical assistance. This work was supported in part by KAKENHI from the Japan Society for the Promotion of Science (JSPS) (to KT; 15KK0343, $15 J 40220$ and HS; 15K15202, 15KK0257, 15H05724). Tissues of Nihonzaru (Japanese macaque monkeys) were provided in part by National Institutes of Natural Sciences (NINS) through the National Bio-Resource Project (NBRP) of the MEXT, Japan. KT, TO and KS are supported by Research Fellowships of JSPS for Young Scientists.

1 Brown DJ, Hill ST, Baker HW. Male fertility and sexual function after spinal cord injury. Prog Brain Res 2006; 152: 427-439.

2 Sipski ML. Sexual functioning in the spinal cord injured. Int J Impot Res 1998; 10(Suppl 2): S128-S130.

3 Breedlove SM. Hormonal control of the anatomical specificity of motoneuron-to-muscle innervation in rats. Science 1985; 227: 1357-1359.

4 Morris JA, Jordan CL, Breedlove SM. Sexual differentiation of the vertebrate nervous system. Nat Neurosci 2004; 7: 1034-1039.

5 Matsuda K, Sakamoto H, Kawata M. Androgen action in the brain and spinal cord for the regulation of male sexual behaviors. Curr Opin Pharmacol 2008; 8 747-751.

6 Breedlove SM, Arnold AP. Hormonal control of a developing neuromuscular system. I. Complete demasculinization of the male rat spinal nucleus of the bulbocavernosus using the anti-androgen flutamide. J Neurosci 1983; 3: 417-423.

7 Breedlove SM, Arnold AP. Hormonal control of a developing neuromuscular system. II. Sensitive periods for the androgen-induced masculinization of the rat spinal nucleus of the bulbocavernosus. J Neurosci 1983; 3: 424-432.

8 Sakamoto $\mathrm{H}$. Brain-spinal cord neural circuits controlling male sexual function and behavior. Neurosci Res 2012; 72: 103-116.

9 Sakamoto $\mathrm{H}$. Sexually dimorphic nuclei in the spinal cord control male sexual functions. Front Neurosci 2014; 8: 184.

10 Sakamoto H, Matsuda K-I, Zuloaga DG, Hongu H, Wada E, Wada K et al. Sexually dimorphic gastrin releasing peptide system in the spinal cord controls male reproductive functions. Nat Neurosci 2008; 11: 634-636.

11 Sakamoto H, Saito K, Marie-Luce C, Raskin K, Oti T, Satoh K et al. Androgen regulates development of the sexually dimorphic gastrin-releasing peptide neuron system in the lumbar spinal cord: evidence from a mouse line lacking androgen receptor in the nervous system. Neurosci Lett 2014; 558: 109-114.

12 Sakamoto H, Takanami K, Zuloaga DG, Matsuda K, Jordan CL, Breedlove SM et al. Androgen regulates the sexually dimorphic gastrin-releasing peptide system in the lumbar spinal cord that mediates male sexual function. Endocrinology 2009; 150 3672-3679

13 Tamura K, Kobayashi Y, Hirooka A, Takanami K, Oti T, Jogahara T et al. Identification of the sexually dimorphic gastrin-releasing peptide system in the lumbosacral spinal cord that controls male reproductive function in the mouse and Asian house musk shrew (Suncus murinus). J Comp Neurol 2017; 525: 1586-1598.

14 Gibbs RA, Rogers J, Katze MG, Bumgarner R, Weinstock GM, Mardis ER et al. Evolutionary and biomedical insights from the rhesus macaque genome. Science 2007; 316: 222-234.

15 Takanami K, Sakamoto H, Matsuda KI, Satoh K, Tanida T, Yamada S et al. Distribution of gastrin-releasing peptide in the rat trigeminal and spinal somatosensory systems. J Comp Neurol 2014; 522: 1858-1873.

16 Satoh K, Takanami K, Murata K, Kawata M, Sakamoto T, Sakamoto H. Effective synaptome analysis of itch-mediating neurons in the spinal cord: a novel immunohistochemical methodology using high-voltage electron microscopy. Neurosci Lett 2015; 599: 86-91.

17 Satoh K, Takanami K, Murata K, Kawata M, Sakamoto T, Sakamoto H. Threedimensional visualization of multiple synapses in thick sections using high-voltage electron microscopy in the rat spinal cord. Data Brief 2015; 4: 566-570.

18 Oti T, Takanami K, Katayama N, Edey T, Satoh K, Sakamoto T et al. Perinatal testosterone exposure is critical for the development of the male-specific sexually dimorphic gastrin-releasing peptide system in the lumbosacral spinal cord that mediates erection and ejaculation. Biol Sex Differ 2016; 7: 4.

19 Oti T, Satoh K, Saito K, Murata K, Kawata M, Sakamoto T et al. Three-dimensional evaluation of the spinal local neural network revealed by the high-voltage electron microscopy: a double immunohistochemical study. Histochem Cell Biol 2012; 138: 693-697.

20 Katayama N, Oti T, Takanami K, Sakamoto T, Sakamoto H. Postnatal development of the gastrin-releasing peptide system in the lumbosacral spinal cord controlling male reproductive function in rats. Proc Jpn Acad Ser B Phys Biol Sci 2016; 92 $69-75$. 
21 Morgan C, Nadelhaft I, de Groat WC. The distribution of visceral primary afferents from the pelvic nerve to Lissauer's tract and the spinal gray matter and its relationship to the sacral parasympathetic nucleus. J Comp Neurol 1981; 201: 415-440.

22 Espinosa-Medina I, Saha O, Boismoreau F, Chettouh Z, Rossi F, Richardson WD et al. The sacral autonomic outflow is sympathetic. Science 2016; 354: 893-897.

23 Vizzard MA, Erdman SL, de Groat WC. Increased expression of neuronal nitric oxide synthase (NOS) in visceral neurons after nerve injury. J Neurosci 1995; 15(5 Pt 2): 4033-4045.

24 Minamino N, Kangawa K, Matsuo H. Neuromedin C: a bombesin-like peptide identified in porcine spinal cord. Biochem Biophys Res Commun 1984; 119: 14-20.

25 Takanami K, Sakamoto H. The gastrin-releasing peptide receptor (GRPR) in the spinal cord as a novel pharmacological target. Curr Neuropharmacol 2014; 12: 434-443.

26 Lebacq-Verheyden AM, Krystal G, Sartor O, Way J, Battey JF. The rat prepro gastrin releasing peptide gene is transcribed from two initiation sites in the brain. $\mathrm{Mol}$ Endocrinol 1988; 2: 556-563.

27 Spindel ER, Chin WW, Price J, Rees LH, Besser GM. Cloning and characterization of cDNAs encoding human gastrin-releasing peptide. Proc Natl Acad Sci USA 1984; 81: 5699-5703.

28 Lemaire S, Trifaro JM, Chouinard L, Cecyre D, Dessureault J, Mercier P et al. Structural identification, subcellular localization and secretion of bovine adrenomedullary neuromedin C [GRP-(18-27)]. Peptides 1989; 10: 355-360.

29 McDonald TJ, Jornvall H, Nilsson G, Vagne M, Ghatei M, Bloom SR et al. Characterization of a gastrin releasing peptide from porcine non-antral gastric tissue. Biochem Biophys Res Commun 1979; 90: 227-233.

30 Fraser M, McDonald TJ, Spindel ER, Fahy M, Hill D, Challis JR. Gastrin-releasing peptide is produced in the pregnant ovine uterus. Endocrinology 1994; 135: 2440-2445.

31 Reeve JR Jr, Walsh JH, Chew P, Clark B, Hawke D, Shively JE. Amino acid sequences of three bombesin-like peptides from canine intestine extracts. J Biol Chem 1983; 258: $5582-5588$.
32 Shaw C, Thim L, Conlon JM. Primary structure and tissue distribution of guinea pig gastrin-releasing peptide. J Neurochem 1987; 49: 1348-1354.

33 Sakamoto H, Arii T, Kawata M. High-voltage electron microscopy reveals direct synaptic inputs from a spinal gastrin-releasing peptide system to neurons of the spinal nucleus of bulbocavernosus. Endocrinology 2010; 151: 417-421.

34 Dobberfuhl AD, Oti T, Sakamoto H, Marson L. Identification of CNS neurons innervating the levator ani and ventral bulbospongiosus muscles in male rats. J Sex Med 2014; 13: 664-677.

35 Forger NG, Breedlove SM. Sexual dimorphism in human and canine spinal cord: role of early androgen. Proc Natl Acad Sci USA 1986; 83: 7527-7531.

36 Breedlove SM, Arnold AP. Hormone accumulation in a sexually dimorphic motor nucleus of the rat spinal cord. Science 1980; 210: 564-566.

37 Sengelaub DR, Forger NG. The spinal nucleus of the bulbocavernosus: firsts in androgen-dependent neural sex differences. Horm Behav 2008; 53: 596-612.

38 Onufrowicz B. Notes on the arrangement and function of the cell groups in the sacral region of the spinal cord. J Nerv Mental Dis 1899; 26: 498-504.

39 Nakagawa S. Onuf's nucleus of the sacral cord in a South American monkey (Saimiri): its location and bilateral cortical input from area 4. Brain Res 1980; 191: 337-344.

40 Sato M, Mizuno N, Konishi A. Localization of motoneurons innervating perineal muscles: a HRP study in cat. Brain Res 1978; 140: 149-154.

41 Sun YG, Chen ZF. A gastrin-releasing peptide receptor mediates the itch sensation in the spinal cord. Nature 2007; 448: 700-703.

42 Ayta IA, McKinlay JB, Krane RJ. The likely worldwide increase in erectile dysfunction between 1995 and 2025 and some possible policy consequences. BJU Int 1999; 84: 50-56.

43 Hehemann MC, Kashanian JA. Can lifestyle modification affect men's erectile function? Transl Androl Urol 2016; 5: 187-194.

44 Briganti A, Salonia A, Gallina A, Sacca A, Montorsi P, Rigatti P et al. Drug Insight: oral phosphodiesterase type 5 inhibitors for erectile dysfunction. Nat Clin Pract Urol 2005; 2: 239-247. 